\title{
Les nouvelles du Roland furieux
}

\section{Giuseppe Sangirardi}

\section{OpenEdition \\ Journals}

Édition électronique

URL : http://journals.openedition.org/cei/171

DOI : $10.4000 /$ cei. 171

ISSN : 2260-779X

\section{Éditeur}

UGA Éditions/Université Grenoble Alpes

\section{Édition imprimée}

Date de publication : 15 mars 2010

Pagination : 115-128

ISBN : 978-2-84310-164-9

ISSN : 1770-9571

Référence électronique

Giuseppe Sangirardi, «Les nouvelles du Roland furieux », Cahiers d'études italiennes [En ligne], 10 | 2010, mis en ligne le 15 septembre 2011, consulté le 27 mars 2021. URL : http://journals.openedition.org/ cei/171 ; DOl : https://doi.org/10.4000/cei.171 


\title{
LES NOUVELLES DU ROLAND FURIEUX
}

\author{
Giuseppe Sangirardi \\ Université de Bourgogne
}

Dans le deuxième chant du Roland furieux, Bradamante, qui vient de désarçonner Sacripant, suit son chemin à la recherche de Roger, dont elle est amoureuse. Elle tombe alors sur un chevalier assis au bord d'une fontaine, à l'air triste et déconfit (II 35, 8 «e si mostrava addolorato e lasso»), et lui demande la cause de sa douleur. Le chevalier prend la parole et la garde pour une vingtaine de strophes (37-57), au cours desquelles se déploie le récit de l'aventure fantastique qui l'a mené jusque-là. La femme qu'il aime lui a été enlevée par un voleur qui monte un cheval ailé et qu'il a en vain suivi jusqu'à son château, construit par les démons sur un rocher inaccessible. C'est également en vain que deux héros arrivés au château au même moment, Gradasse et Roger, lui ont offert leur secours, car ils n'ont rien pu contre les armes magiques du voleur : le chevalier - dont on apprend plus tard qu'il est Pinabel de Mayence - est reparti en proie au désespoir. On devine aisément l'intérêt que trouve Bradamante à écouter le récit de Pinabel, qui fait surgir inopinément l'image de l'être aimé dont elle est en quête : intérêt qui sert de miroir au plaisir que prend le lecteur de l'Arioste à cette narration pleine de surprises (entre autres, celle d'assister à la première apparition de l'hippogriffe, et celle de retrouver le fil d'un épisode interrompu à la fin du Roland amoureux de Boiardo, où Gradasse et Roger avaient suivi un nain qui les avait amenés à un château, III VII 55).

Or, l'artifice narratif qui construit cette petite chambre des merveilles est celui du récit intercalé au second degré (ou «métadiégétique» dans le langage de la narratologie). En cédant momentanément la parole à un personnage, l'auteur crée un circuit narratif interne au récit, dans lequel le lecteur peut reconnaitre le reflet de sa propre expérience de lecture. L'auteur crée en même temps un double de sa propre image sur lequel il peut se projeter autrement que sur l'image du narrateur "premier», ainsi qu'un espace thématique ouvert, où apparaissent des formes plus libres, 
car moins liées par les lois de la cohérence aux conventions établies dans le récit du narrateur.

Le récit de Pinabel n'est pas un cas isolé dans le Roland furieux: il n'est, au contraire, que la première d'une série de narrations au second degré qui, dans la tradition critique, ont reçu le plus souvent le titre de nouvelles. Le corpus des nouvelles, identifié pour l'essentiel dans les appareils critiques et érudits des anciennes éditions ${ }^{1}$, est toutefois sujet à des fluctuations, qui sont celles mêmes de ses critères de définition, longtemps restés implicites. Même quand les critères pour identifier les nouvelles ont été énoncés, ils n’ont pas toujours été identiques, et ils n’ont donc pas donné les mêmes résultats : dans la perspective narratologique de G. Dalla Palma, les nouvelles, qu'il appelle plus souvent diversioni (car elles constituent une digression dans le parcours des héros), sont quatorze ${ }^{2}$; pour A. Franceschetti, qui précise le critère de définition et propose d'identifier les nouvelles aux récits de personnages dont l'intrigue est achevée en soi et secondaire par rapport à l'action principale, le catalogue comporte treize entrées ${ }^{3}$. Quant à moi, je retiens comme critère essentiel la présence d'un récit métadiégétique, dont la longueur soit suffisante pour y reconnaître les lignes d'une intrigue, mais aussi la taille moyenne de la nouvelle en tant que genre codifié : ma liste comprend ainsi quinze nouvelles, dont douze présentes dès la première édition (I5I6) et trois ajoutées dans la dernière $(\mathrm{I} 532)^{4}$.

Outre la différence des points de vue, c'est la variété intrinsèque du corpus qui peut expliquer ces hésitations de lecture. Rapprochées par leur statut métadiégétique commun, les nouvelles se différencient par nombre d'autres caractéristiques : l'attitude du narrateur et la fonction de son récit par rapport à la situation des personnages et par rapport à la construction du roman; puis la taille du récit, son contenu et ses modèles, pour ne citer que les principales. La variété de la morphologie des nouvelles renvoie aussi, en effet, à celle de leur généalogie littéraire. Car, non seulement l'Arioste n'est évidemment pas l'inventeur du récit intercalé (dont on trouve des antécédents dans la littérature grecque et latine ancienne, et

I. A. Franceschetti, "La novella nei poemi del Boiardo e dell'Ariosto", dans La novella italiana, Atti del Convegno di Caprarola (I9-24 septembre I988), Rome, Salerno Editrice, I989, 2 vol., t. II, p. 8I4-8I6.

2. G. Dalla Palma, Le strutture narrative dell'Orlando furioso, Florence, Olschki, 1984, p. I39.

3. A. Franceschetti, «La novella», art. cité, p. 819-820 et p. 828-829.

4. Voir G. Sangirardi, Ludovico Ariosto, Florence, Le Monnier, 2006, p. I26. La différence de mon critère par rapport à ceux de Franceschetti et Dalla Palma tient, me semble-t-il, au fait que je ne prends pas en compte le caractère digressif de la nouvelle, le changement de narrateur me semblant un élément à lui seul susceptible de déclencher le glissement générique. 
notamment chez Ovide, l'un des inspirateurs des nouvelles de l'Arioste)', mais il n'est pas non plus le premier qui l'ait adopté dans un roman de chevalerie.

L'inscription de la nouvelle dans le corps du roman chevaleresque remonte aux grandes compilations en prose d'oïl du XIII ${ }^{\mathrm{e}}$ siècle, du moins si l'on s'en tient à la définition formelle de la nouvelle que j'ai donnée ci-dessus (récit métadiégétique d'une certaine longueur). En effet, l'artifice métadiégétique est largement présent dans le Tristan en prose et surtout dans Guiron le courtois. Dans ce dernier livre, le réseau des récits métadiégétiques constitue même la véritable ossature de l'édifice romanesque et, d'après l'analyse qu'en a fournie R. Lathuillère ${ }^{6}$, une source très riche d'effets narratifs brillants. Les chevaliers du Guiron passent beaucoup de temps à se raconter des histoires, qu'il s'agisse d'expliquer l'origine d'une coutume ou la cause d'une aventure, ou encore de se remémorer leur passé. Les héros de ces contes au second degré, quand ils ne relatent pas le vécu du narrateur, sont tantôt lointains, tantôt ceux-là mêmes qui les écoutent, à l'insu du narrateur; les récits sont parfois interrompus et puis recommencés, et il arrive que l'auditeur en sache plus que le narrateur. Les jeux de miroir, les reconnaissances inattendues, les dévoilements surprenants du genre que nous avons évoqués à propos du récit de Pinabel, et qui se retrouvent souvent dans les nouvelles de l'Arioste, abondent dans Guiron et ce n'est donc certainement pas un hasard si cinq des quinze nouvelles du Roland furieux ont leur modèle principal dans un récit métadiégétique du Guiron?.

Si le récit intercalé est un artifice narratif fondamental dans le Guiron, au service notamment de la manipulation du temps et de la perspective, il serait toutefois plus incertain d'y voir une pratique de «modulation»

5. L'histoire de Procris et Céphale, racontée par Ovide dans les Métamorphoses (VII 690-865), sert de modèle à la nouvelle du juge Anselme (XLIII 69-I43), bien qu'avec le concours d'autres sources parallèles (voir P. Rajna, Le fonti dell'Orlando furioso, réimpr. anastatique de la $2^{\mathrm{e}}$ éd. de 1900, Florence, Sansoni, 1975, p. 581 et suiv.). Par ailleurs, on peut rappeler que la nouvelle de Pirame et Thisbé (Mét., IV 53-I66), à l'origine de la nouvelle de Prasildo, Iroldo et Tisbina dans le Roland amoureux de Boiardo (I xII 4-89), aurait aussi inspiré une tragédie juvénile de l'Arioste, selon le témoignage des anciens biographes.

6. R. Lathuillère, «Un exemple de l'évolution du roman arthurien en prose dans la deuxième moitié du XIII ${ }^{\mathrm{e}}$ siècle", dans Mélanges de langue et littérature françaises du Moyen Âge offerts à Pierre Jonin, Aix-enProvence, Publications du CUERMA, Université de Provence, 1979, p. 387-40I; Idem, "L'évolution de la technique narrative dans le roman arthurien en prose au cours de la deuxième moitié du XIII ${ }^{\mathrm{e}}$ siècle», dans Études de langue et de littérature françaises offertes à André Lanly, Université Nancy 2, 1980, p. 203-2I4.

7. Il s'agit des histoires racontées par Pinabel (ch. II), Isabelle (ch. XIII), Ermonide (ch. XXI), Lydie (ch. XXXIV) et par l'hôte du château de Tristan (ch. XXXII); pour les épisodes correspondants du Guiron, voir Rajna, 1975. À cela il faudrait ajouter deux nouvelles (celle de Dalinde, ch. V, et celle de Marganor, ch. XXXVII) pour lesquelles le Guiron fournit des suggestions mineures (voir toujours Rajna, 1975). 
générique $^{8}$, la «citation» du genre de la nouvelle, qui à l'époque n'était pas vraiment reconnu en tant que tel. Les formes narratives qui constituent la préhistoire de la nouvelle, tel le fabliau ou l'exemplum, ne sont pas spécialement évoquées par les récits des chevaliers : en réalité, l'univers thématique des récits intercalés du Guiron ne diffère pas de celui de la narration au premier degré, et ne donne pas lieu à un glissement générique évident.

Mais les changements intervenus dans l'histoire littéraire entre le siècle du Guiron et le temps du Roland furieux imposent une lecture différente des courbes métadiégétiques qui se profilent dans le dessin narratif de l'Arioste. C'est notamment l'avènement du Décaméron sur la scène littéraire européenne qui à la fois impose la nouvelle en tant que genre et lui donne une image caractérisée. L'un de ses traits distinctifs devient justement, grâce à la présence de la cornice (récit cadre), le statut métadiégétique; un autre, sa fonction pragmatique promue (l'acte de raconter des histoires, pour le plaisir, mais aussi pour en tirer des leçons, comme c'était déjà le cas dans les exempla des prédicateurs, a gagné en légitimité et en résonance). Désormais, tout récit métadiégétique, raconté pour le plaisir ou pour fournir des modèles moraux, ne peut plus échapper au soupçon d'être une nouvelle. Un récit métadiégétique intercalé dans un roman, après Boccace, demande à être reconnu - du moins par hypothèse préliminaire et quitte à ce que la lecture suggère d'autres interprétations comme une nouvelle.

On voit ce changement à l'œuvre dans le Roland amoureux de Boiardo et dans le Mambrien du Cieco de Ferrare, qui tous les deux, avant l'Arioste, introduisent quelques narrations au second degré dans leur roman. Dans le Roland amoureux on a compté sept narrations métadiégétiques ${ }^{9}$, de tailles diverses; quatre d'entre elles sont introduites par une référence explicite au genre de la novella: par exemple, la plus connue, qui raconte l'histoire de Prasildo, Iroldo et Tisbina ( $A$ Acciò che men te incresca il caminare / Per questa selva horribil e diserta, / Una novella te voglio contare»,

8. Pour la notion de modulation générique voir A. Fowler, Kinds of Literature. An Introduction to the Theory of Genres and Modes, Cambridge MA, Harvard University Press, 1982.

9. Dont deux «doubles ", car reprises ou prolongées après une première conclusion (cf. les histoires de Prasildo, Iroldo et Tisbina, I xir 3-89 et XVII 2-I6, et de Leodilla, I xxi 49-69 et Xxi Io-46). Pour la liste complète, voir Franceschetti, «La novella», art. cité, p. 822, mais il faudrait préciser que la sixième des sept nouvelles indiquées par Franceschetti (celle de Narciso et Silvanella, II XVII 50-6I) est racontée par le narrateur, mais ensuite attribuée à Calidora (II XVII 63, I-3) : distraction de l'auteur, peut-être, mais plus vraisemblablement jeu malicieux, par lequel il transgresse et rétablit à la fois un code narratif en train de se constituer. 
I XII 4, I-3) $)^{\text {Io }}$. Parfois, l'étiquette générique est assortie d'une motivation pragmatique qui renvoie également à l'univers de la nouvelle : on raconte une histoire pour remplir les temps morts d'un voyage (I XII 4, I et II XXVI 20, 8; on pense naturellement aux Canterbury Tales) ou pour consoler quelqu'un de sa souffrance amoureuse (I XXI 48, 6-8; "Umana cosa è aver compassione degli afflitti», on le sait, est la première phrase du Décaméron, qui se donne pour mission prioritaire de soulager les peines d'amour de ses lectrices). Surtout, les recueils de nouvelles apparaissent désormais dans le catalogue des sources (le Décaméron dans la nouvelle de Prasildo, Iroldo et Tisbina, le Libro dei Sette Savi dans la seconde partie de l'histoire de Leodilla), tandis que dans deux narrations (celles de Leodilla et celle de Doristella) émerge le thème de la beffa de la femme contre le mari jaloux, inscrit définitivement par les VII et $\mathrm{VIII}^{\mathrm{e}}$ journées du Décaméron au répertoire caractéristique de la nouvelle. Cependant, si Boiardo mise parfois sur des effets de friction entre le genre romanesque et la nouvelle, il hérite également des procédés du récit intercalé propres aux romans comme Tristan et Guiron: la narration peut être introduite comme explication d'une coutume ou de la situation d'un personnage ${ }^{\text {II, }}$ et les personnages et les thèmes des nouvelles ne marquent pas, le plus souvent, de véritables solutions de continuité par rapport à la narration au premier degré, y compris quand les modèles viennent de la littérature et de la mythologie anciennes. Le travestissement romanesque, en d'autres termes, sert de contrepoids efficace aux effets de citation de la nouvelle comme genre distinct : celui-ci semble à la fois ostensiblement reconnu et habilement mimétisé, avec un double effet de modulation générique réciproque (la nouvelle modifie le roman, le roman modifie la nouvelle). Le phénomène le plus symptomatique de cette logique d'échange est la circulation des personnages entre la nouvelle et le roman : il y a ceux qui racontent leur propre histoire et sont ainsi dès l'origine des personnages du roman, et ceux qui «sortent» d'une nouvelle racontée par un tiers et se retrouvent de manière inattendue parmi les héros de la chevalerie (c'est le cas de Prasildo et Iroldo).

Io. Voir aussi les nouvelles d'Albarosa, Polindo et Trufaldino, où il s'agit d'ailleurs de la lecture d'un livre (I XIII 29, 5-6 : "Quel libro a chi lo legie dichiarava/ Tutta la historia e la novella scura»), de Leodilla (I xxi 69, 6 : «la mia novella voglio seguitare» : l'étiquette générique est ici introduite au moment de la suspension du récit) et de Doristella (II xxvi 20, 6-8: «Ch’io sia colei che con qualche novela/ Facia trovar l'albergo più vicino, / Perché parlando, s'ascorta il camion "; et 53, 3 : "Ché non era compita sua novella " : l'étiquette est ici placée au début et à la fin, celle-ci étant d'ailleurs donnée comme ouverte).

II. Voir les nouvelles de Stella et Marchino (I viII 27-52), d'Origille (I xxix 5-35), de Narcisse (II XviI 50-6I) et la nouvelle «écrite» de Polindo et Albarosa (I XIII 30-45). 
L'effet de friction entre genres est, en revanche, nettement plus sensible dans le Mambrien. Les sept nouvelles introduites dans celui-ci ${ }^{12}$ sont inscrites dans une cornice (banquet festif ou voyage), ou tout au moins racontées dans le but explicite d'offrir un exemple moral à méditer : en aucun cas il ne s'agit d'un récit d'aventures vécues par le narrateur ou ses pairs, comme dans le modèle du récit intercalé romanesque ${ }^{\mathrm{r} 3}$, et à deux reprises le narrateur est une figure professionnelle (voir les histoires racontées aux banquets de Renaud et Carandine, II 42-II5, et de Feburro et Fulvia, $X$ 5-59). D'ailleurs, les récits sont régulièrement clos par une morale qui renvoie à la préhistoire de la nouvelle (la littérature des exempla), tandis que les thèmes (l'astuce et la tromperie déployées à des fins érotiques, les rapports houleux entre maris et femmes, la jalousie et la beffa, etc.) et les modèles semblent solidement ancrés dans la tradition des fabliaux et des contes populaires ${ }^{14}$. Malgré le travestissement romanesque qui opère dans la versification et dans l'adaptation des noms, des lieux et des circonstances, la greffe de la nouvelle sur le corps du roman est plus que visible : elle est exhibée, sans doute dans le but de rendre reconnaissable au public un code narratif séduisant, là où les ressources du régime romanesque semblent faire défaut à un auteur en difficulté. Quelles qu'aient pu être les difficultés du Cieco de Ferrare, d'ailleurs, sa pratique désinhibée du collage générique est aussi un témoignage à la fois de la place grandissante que la nouvelle occupe dans le panorama des genres reconnus ${ }^{15}$, et de son installation dans le répertoire des genres susceptibles d'être phagocytés par le grand cannibale, le roman d'armes et d'amour, non sans produire une réaction propre à affecter le métabolisme du dévorateur.

Arrivant à ce point de l'histoire, l'Arioste double la mise et gagne une place légitime ${ }^{16}$ dans l'histoire de la nouvelle. La place qu'occupent les nouvelles, déjà considérable dans le Mambrien, devient importante dans le Roland furieux : 650 strophes dans la dernière édition, soit presque un huitième du volume total de l'œuvre. Ce n'est certainement pas assez

I2. Elles ont été étudiées par G. Rua, Novelle del Mambriano del Cieco da Ferrara esposte e illustrate, Turin, Loescher, I888.

13. L'exception de la nouvelle racontée par Maugis (II 36-76) n'est qu'apparente : celui-ci se présente à Carandine sous les faux-semblants d'un naufragé grec, dont il prétend ensuite raconter l'histoire : le lecteur, lui, sait qu'il ment et reconnaît aisément le profil thématique d'une nouvelle.

I4. Cependant, la dernière des narrations au second degré du Mambrien, très longue, masque sous ses allures de nouvelle l'intrigue d'un roman à la manière alexandrine : nouveau signe des échanges et de modulations possibles entre les formes du roman et celles de la nouvelle.

I5. Elle arrivera à être codifiée peu après le roman, tout en restant plus marginale, en raison de sa plus grande distance du genre narratif le plus prestigieux, l'épopée.

I6. Reconnue d'abord par les premiers théoriciens italiens de ce genre : voir à ce sujet N. Ordine (éd.), Traités sur la nouvelle à la Renaissance. Bonciani, Bargagli, Sansovino, Paris-Turin, Vrin-Aragno, 2002. 
pour que l'on considère l'action du roman comme une cornice pour les nouvelles qu'il contient, mais c'est suffisant pour que l'on cherche le sens de cette interférence manifeste de la nouvelle sur le roman. S'interrogeant justement sur l'interaction des deux genres, la nouvelle et le roman, dans le Roland furieux, un critique récent ${ }^{17}$, a mis en exergue à sa réflexion la citation suivante de M. Bakhtine :

Le roman parodie les autres genres (justement, en tant que genres); il dénonce leurs formes et leur langage conventionnels, élimine les uns, en intègre d'autres dans sa propre structure en les réinterprétant, en leur donnant une autre résonance ${ }^{18}$.

Lire la pratique du récit au second degré comme une manifestation de l'attitude typique du roman à l'égard des autres genres implique non seulement, comme le suggère Bigazzi, que l'on soit certain que le Roland furieux est un roman, mais plus exactement, que l'on croie que la notion bakhtinienne de roman lui convienne. Bakhtine, comme on le sait, a vu dans le roman le genre de la transgression et du devenir, le genre dont la règle principale est de transgresser les règles et les limites, d'incarner le mouvement perpétuel de la vie, un éternel présent toujours ouvert, qui s'oppose à l'immobilité des règles définies et du passé; bref, plutôt un anti-genre qu'un genre, héritier légitime de la notion schlegelienne de roman comme ouvre romantique par excellence, car forme de création incessante et illimitée. Quand on se souvient que les romans de chevalerie étaient l'un des modèles semblant incarner cet idéal aux yeux de Friedrich Schlegel, on voit l'intérêt qu'on peut trouver à appliquer la théorie de Bakhtine au roman de l'Arioste. Mais la notion bakhtinienne de genre, si séduisantes que soient ses implications anthropologiques et si pertinente que puisse paraître son application aux opérations parodiques et ironiques de l'Arioste, présente l'inconvénient, ou tout au moins la caractéristique, d'être l'une de ces catégories "extrinsèques" (Hirsch) ou "exogènes» (Schaeffer), instituées par des théoriciens dans le but de classer les œuvres, sans prendre en compte nécessairement les filiations historiques et les intentions des auteurs. Nous pouvons, bien sûr, trouver ces classements satisfaisants, et nous les approprier. Mais il est au moins aussi légitime de chercher à reconnaître un projet générique d'auteur, autrement dit, ce que, d'après Hirsch, on appelle le genre intrinsèque.

17. R. Bigazzi, "Le novelle del Furioso", Riscrittura Intertestualità Transcofidicazione. Personaggi e scenari, Seminario di studi (Pise, février-mai 1993), E. Scarano et D. Diamanti (dir.), Pise, Tipografia Editrice Pisana, 1994, p. 47-57.

18. La citation, que Bigazzi propose dans la traduction italienne, est tirée de M. Bakhtine, Esthétique et théorie du roman, Paris, Gallimard, 1978, p. 443. 
Or, si nous essayons d'identifier l'intention générique de l'Arioste, en déchiffrant les signaux multiples et plus ou moins conscients qu'il a déposés dans son texte (le titre, les déclarations du proème, le choix de la forme métrique et narrative, celui de l'univers des personnages), nous pouvons sans doute convenir que le Roland furieux est un roman. Cette décision demande en réalité à être étayée par une argumentation complexe, mais nous pouvons ici nous contenter d'un raisonnement simplifié. En effet, des deux profils génériques entre lesquels le Roland furieux et surtout ses lecteurs semblent hésiter, le roman et l'épopée, c'est le premier et non le second qui constitue la référence fondamentale de l'Arioste. Il est vrai que le roman de chevalerie, notamment dans la version qui compte le plus pour l'Arioste, celle qu'en a donnée Boiardo, intègre des éléments de l'épopée, à la fois classique et médiévale. Mais, pour reprendre le langage de Fowler, cela ne représente qu'un effet plus ou moins accentué de modulation. Il est vrai, également, que l'Arioste introduit dans le Roland furieux des traits qui renvoient à l'épopée ancienne plus manifestement que ne l'avait fait Boiardo : mais cette correction plus vigoureuse de la trajectoire romanesque ne remet pas en question la direction fondamentale de celle-ci. Faire de l'épopée à l'époque de l'Arioste ne pouvait vouloir dire autre chose qu'imiter l'Enéide de Virgile (Bembo et Vida n'ont aucun doute sur ce point), mais l'imitation virgilienne dans le Roland furieux n'est pas le choix décisif de l'Arioste; son choix décisif, c'est de continuer le roman de Boiardo, et l'imitation de l'épopée ancienne, accrue mais déjà présente dans son modèle, n'est qu'une coloration modale (si forte soit-elle) qui vient caractériser le genre romanesque adopté.

Or, si l'on peut dire que l'Arioste a choisi le roman, ce qui nous importe c'est surtout la nature de sa décision générique. Dans la perspective de Bakhtine, le roman est un anti-genre, ce qui suppose à la fois l'existence antérieure d'un système générique prescriptif, et le désir de le transgresser : le pathos que le romantisme puis Bakhtine accordent à la liberté romanesque n'est que la conséquence de la contrainte longtemps imposée par la réglementation classiciste de la littérature. Mais à l'époque de l'Arioste, ce pathos ne peut pas exister sous la même forme, puisqu'il n'existe pas encore une codification des genres prescriptive ou simplement dominante. C'est juste après la dernière édition du Roland furieux, comme on le sait, que la dispute sur les genres, inspirée par la lecture de la Poétique d'Aristote, envahit la scène littéraire et dramatise les choix génériques des poètes (c'est alors que le Roland furieux devient, rétrospectivement, un enjeu important de ce débat). Avant cette éclosion du classicisme des règles, la culture humaniste dans laquelle l'Arioste s'était formé n'ignorait 
certainement pas l'existence des genres, mais ceux-ci, identifiés et classés de manière empirique, constituaient simplement la toile de fond sur laquelle s'inscrivait la théorie bien plus cruciale de l'imitatio, avec la problématique du choix d'un ou de plusieurs auctores ${ }^{19}$. Au cœur des mécanismes de la filiation littéraire, en d'autres termes, il y avait l'imitation du style d'un auctor, et non le respect des règles d'un genre (comme ce sera le cas au milieu du $\mathrm{XVI}^{\mathrm{e}}$ siècle); c'est donc dans la figure de l'auctor que se cristallise essentiellement le principe d'autorité en littérature, et c'est contre cette figure que se dirigent surtout les intentions transgressives, le "genre» ne jouant pas encore un rôle crucial dans la symbolique et dans l'idéologie littéraires, et pouvant donc rester empirique et indéterminé. Ainsi, s'il est vrai que l'ironie de l'Arioste - son insoumission aux règles et à l'autorité, sa posture manipulatrice et ludique, son conflit odipien assez net avec la tradition - s'exerce incessamment à l'égard de la parole des auctores, il serait sans doute anachronique de lui prêter le programme bakhtinien de jouer avec les genres, ou en tout cas de le faire avec une conscience et un pathos qui ne peuvent être concevables quaprès le romantisme. La partie d'indéterminé et d'ambigu qu'il y a dans les profils génériques du Roland furieux, si elle traduit pour une part l'attitude manipulatrice de l'Arioste face au langage des pères littéraires, d'un point de vue strictement générique se situe plutôt en deçà qu'au-delà de l'établissement de la règle.

Mais il y a aussi une autre raison, peut-être plus décisive que cette première, qui rend peu productif le modèle herméneutique bakhtinien pour la lecture des nouvelles du Roland furieux. En effet, non seulement les précédents constitués par Boiardo et par le Cieco de Ferrare faisaient de la nouvelle un élément en partie déjà intégré dans le répertoire romanesque, mais dans la dialectique entre citation et mimétisation de la nouvelle instaurée par ses prédécesseurs, l'Arioste avance décidément dans le sens de la mimétisation. S’il reste dans les récits métadiégétiques du Roland furieux plusieurs éléments qui maintiennent la référence au genre de la nouvelle, par rapport non seulement au Mambrien, mais aussi au Roland amoureux, les procédés de travestissement et d'intégration dans le roman prennent largement le dessus. L'Arioste, d'abord, n'adopte jamais

19. Cela vaut pour Bembo (voir Sangirardi, Ludovico Ariosto, op. cit., p. II9-I20) comme pour Vida (qui dans son Art poétique commence par évoquer la différence des genres, mais construit la suite de ce traité comme s'il n'y avait qu'un seul modèle de poésie, l'épopée et Virgile). 
l'étiquette générique la plus explicite ( novella») ${ }^{20}$. Les situations-cadre typiques de la nouvelle - le voyage, la fête, le banquet ou la réunion d'un groupe évoquant la brigata du Décaméron - ne se retrouvent que dans une minorité de $\operatorname{cas}^{21}$. Dans certains d'entre eux, d'ailleurs, les modalités ou le contenu du récit ne renvoient pas spécialement à la tradition de la nouvelle : le motif de l'explication d'une situation de difficulté ${ }^{22}$ ou d'une coutume chevaleresque, par exemple, évoque manifestement le récit intercalé romanesque ${ }^{23}$, et il en va de même pour le statut homodiégétique ${ }^{24}$ ou autodiégétique ${ }^{25}$ de la narration, la nouvelle étant typiquement hétérodiégétique. Il n'y a, en réalité, que deux narrations dans lesquelles le signal de fréquence de la nouvelle soit émis avec netteté : l'histoire de Fiammetta racontée par un aubergiste à Rodomont (XXVIII 4-74) comme exemple d'infidélité et de ruse féminines (où l'on trouve à la fois le narrateur hétérodiégétique, le contexte convivial, le but consolateur et le statut exemplaire du récit, la discussion de la brigata qui l'interprète et les thèmes typiques de la tromperie et de l'érotisme grivois) et l'histoire du juge Anselme qu'un nocher raconte à Renaud (XLIII 69-I43) pour lui prouver que les hommes ne sont pas moins infidèles que les femmes et qu'il a eu raison de ne pas boire à la coupe enchantée qui lui aurait révélé si sa femme était ou non fidèle (où l'on retrouve également des signes génériques forts : le récit de voyage, son statut exemplaire, la thématique de la tromperie et de la jalousie, même si cette fois déclinée dans un registre plus hybride, qui mélange les modèles de la nouvelle et de la mythologie $)^{26}$. Mis à part ces cas, l'Arioste préfère brouiller les pistes et minimiser le heurt des codes génériques ${ }^{27}$.

20. On trouve toutefois "conto intero" à propos de la nouvelle de Marganor (XXXVII 44, 4) et "esempio" par référence à l'histoire de Fiammetta et à celle du chevalier de Mantoue (voir Franceschetti, "La novella", art. cité, p. 8II-8I2).

2I. Pour le voyage, on a les nouvelles de Dalinde (V 5-74), de Richardet et Fleurdépine (XXV 22-70) et du juge Anselme (XLIII 69-I43); la réunion conviviale est le contexte des nouvelles de Norandin (XVII 25-68), de l'île des femmes homicides (XX 5-64), du château de Tristan (XXXII 83-94) et du chevalier de Mantoue (XLIII 9-46).

22. Dans les nouvelles de Dalinde, de Ricciardetto et de l'île des femmes homicides.

23. C'est le cas dans les nouvelles de Norandin et du château de Tristan.

24. Guidon le Sauvage est présent dans l'histoire des femmes homicides qu'il raconte.

25. Richardet est le protagoniste de l'histoire qu'il raconte à Roger; pour Dalinde, qui a un rôle important mais non crucial dans la nouvelle qu'elle raconte, on peut hésiter entre le statut homodiégétique et autodiégétique.

26. On pourrait y ajouter le premier volet du diptyque de nouvelles du chant XLIII, où il y a, en plus du contexte convivial, la même thématique érotique à cheval sur le mythe et la nouvelle : mais dans ce cas, la présence d'un narrateur autodiégétique contribue à atténuer l'effet de citation du genre nouvelle.

27. Il est significatif (comme l'a observé Franceschetti, «La novella», art. cité, p. 836) que même lorsque le cadre du récit permettrait de quitter le monde chevaleresque, comme dans la nouvelle infernale de Lydie 
La mimétisation romanesque des nouvelles, largement dominante dans le Roland furieux, y est assurée principalement par deux options liées entre elles : celle du statut homodiégétique ou (plus souvent) autodiégétique de la narration et celle de l'absorption de l'intrigue de la nouvelle dans l'intrigue du roman. Ce deuxième aspect a déjà été souligné par la critique : G. Dalla Palma avait remarqué que le type dominant est celui de la connexion "syntagmatique» entre la nouvelle et le récit au premier degré, selon lequel l'histoire racontée par un personnage reste suspendue sur une situation injuste, le héros qui l'écoute intervenant ensuite pour rétablir la justice et dénouer l'intrigue ${ }^{28}$. L'articulation narrative préférée par l'Arioste - un héros rencontre un personnage en difficulté qui lui raconte son histoire, puis intervient dans cette histoire pour réparer les torts qui y sont commis - permet à la fois d'intégrer l'univers de la nouvelle dans celui du roman, de la présenter comme une action interne au récit romanesque (le personnage raconte son histoire pour demander de l'aide) et de prolonger le temps du suspense en retardant le dénouement (pendant que le personnage raconte et même à la fin de son récit, on ignore quelle sera l'issue) ${ }^{29}$. Mais la tension dont la nouvelle se voit ainsi chargée est également le fruit du statut du récit, de l'implication personnelle du narrateur dans l'aventure, fait qui a moins attiré l'attention de la critique. Or ce fait est, au contraire, crucial dans l'économie des récits métadiégétiques du Roland furieux. Il l'est d'abord pour des raisons statistiques, car deux tiers des nouvelles sont affectées par ce trait ${ }^{30}$, qui renvoie à la tradition du récit intercalé romanesque et montre que l'Arioste revient en arrière, par rapport au Cieco et à Boiardo, dans la direction du Guiron. Mais si le fait que les chevaliers racontent le plus souvent leur propre histoire nous montre le penchant de l'Arioste pour des nouvelles bien mimétisées dans le récit romanesque, il nous aide aussi à comprendre la logique d'une citation générique à la fois pratiquée et camouflée. Car, si nous avons pu constater

(qui aurait pu facilement se dérouler dans un milieu différent), l'Arioste opte pour la cohérence de l'univers romanesque.

28. G. Dalla Palma, Le strutture, op. cit., p. I4-Is et I4I et suiv.

29. Seulement dans la nouvelle de Richardet l'Arioste fait d'abord intervenir le héros, pour ensuite faire entendre le récit du personnage sauvé (comme c'était le cas chez Boiardo dans la nouvelle de Prasildo et Iroldo et le Cieco dans celle d'Orio et Pulicasta), séquence qui relègue la nouvelle au statut de "passetemps». Le cas de la nouvelle de Dalinde est ambigu, car si celle-ci raconte son histoire après avoir été sauvée par Renaud, son récit ouvre une autre perspective d'intervention héroïque pour le paladin, au bénéfice de Guenièvre et d'Ariodant.

30. On peut qualifier d'autodiégétiques les nouvelles de Pinabel (II 37-57), d'Astolphe (VI 3I-53), d'Olympie (IX 22-56), d'Isabelle (XIII 3-3I), d'Hermonis (XXI II-66), de Lydie (XXXIV II-43) et du chevalier de Mantoue (XLIII 9-46); à celles-ci s'ajoutent les nouvelles plutôt homodiégétiques de Dalinde (V 5-74), de Guidon (XX 5-64) et de la femme exilée par Marganor (XXXVII 38-85). 
que l'Arioste mise sur le travestissement et l'intégration romanesque de la nouvelle, il y a lieu de se demander finalement à quelles fins il use d'un répertoire générique dont il cache en même temps la présence.

On pourrait d'abord répondre à cette question que l'Arioste est toujours à l'aise dans l'espace furtif de l'allusion plutôt que dans celui de la citation, la manipulation ludique et le vol exhibé étant une expression fondamentale de son rapport à la tradition littéraire. Mais cela laisse de toute façon ouverte la question de savoir pourquoi il «vole» précisément des nouvelles dans les armoires de la littérature. Or, si nous regardons à la fois les contenus des nouvelles et la modalité de leur récit, nous observons que leur apport tient pour l'essentiel à une modulation élégiaque du code romanesque. L'Arioste ne profite pas vraiment de l'ouverture de l'espace de la nouvelle pour sortir du répertoire thématique romanesque (codifié par la tradition chevaleresque et notamment par Boiardo), mais plutôt pour proposer de celui-ci une palette restreinte, qui exclut la couleur épique et se concentre sur l'exploration de la relation sentimentale entre les donne et les cavalieri, sur la guerre des cœurs plutôt que sur celle des cuirasses. Le récit à la première personne (d'un personnage qui raconte son histoire) est alors le signe d'un investissement lyrique de cette matière, plus prononcé que d'habitude. Plus précisément, il s'agit d'élégie : dans tous ces cas, celui qui raconte est un personnage en état de souffrance, dont le récit est souvent introduit et accompagné par des larmes ou prononcé avec une voix triste et affaiblie ${ }^{31}$. La voix qui raconte les nouvelles est donc souvent, dans le Roland furieux, une voix éplorée, entrecoupée de sanglots, celui qui raconte est un narrateur englué dans la souffrance sentimentale, dans le pathétique (bien que parfois allégé par les résonances parodiques du vers et de la langue de l'Arioste). Dans des cas extrêmes, qui poussent vers le tragique le ton habituel de l'élégie, le narrateur est mourant (Hermonis) ou déjà mort (Lydie et Astolphe - encore que pour ce dernier la tragédie, en réalité temporaire, soit mêlée de parodie). Les histoires de Lydie et d'Astolphe, d'ailleurs, qui parlent d'un au-delà renvoyant à Virgile et surtout à Dante, montrent à l'évidence que la tonalité élégiaque des récits des chevaliers peut aussi se charger d'allusions «infernales», leurs souvenirs douloureux pouvant faire écho à ceux qui hantent les damnés de la

3I. Par exemple, Pinabel est trouvé par Bradamante figé dans une évidente posture mélancolique (II 35), Dalinde, qui vient d'échapper à la mort, raconte son histoire "con umil voce» (IV 72, 7), tout comme la plante de myrte dans laquelle a été transformé Astolphe, qui s'exprime "con mesta e flebil voce» : les même signes on les retrouve explicitement dans les cas d'Olympie, d'Isabelle et du chevalier de Mantoue. 
Commedia dantesque ${ }^{32}$. Dans ces nouvelles élégiaques, qui sont les plus nombreuses et les plus typiques du Roland furieux, l'Arioste s'empare d'un trait caractéristique du genre novella, le récit au second degré, non pas pour suivre le profil typiquement comique de la nouvelle italienne ${ }^{33}$, mais au contraire pour créer des doubles lyriques du narrateur romanesque. S’il est vrai, comme j'ai essayé de le montrer ailleurs ${ }^{34}$, qu'un poète élégiaque se cache sous l'armure du narrateur ironique du roman, voix d'une douleur enfantine avec laquelle l'Arioste adulte veut jouer pour atteindre le statut paternel, les narrateurs des nouvelles laissent le plus souvent émerger cette élégie secrète, ou tout au moins la poussent vigoureusement vers la surface de l'écriture. Ainsi, dans le Roland furieux la rencontre entre la nouvelle et le roman aboutit à la création d'un espace nouveau, tant pour la nouvelle que pour le roman.

On peut sans doute voir un cas emblématique de cette rénovation de l'espace narratif dans l'avant-dernière des nouvelles de l'Arioste, l'histoire que le chevalier de Mantoue raconte à Renaud après que celui-ci a refusé de boire à la coupe enchantée pour s'assurer de la fidélité de sa femme (XLIII 9-46). Regrettant amèrement de ne pas avoir su se retenir à son tour, le chevalier raconte comment, incité par la magicienne Mélisse, il mit à l'épreuve la fidélité de sa femme en poussant celle-ci à accomplir par dépit la trahison dont il la soupçonnait. Cette narration, sur un sujet des plus typiques de la nouvelle (la jalousie et la tromperie), intervient également dans un contexte convivial caractéristique (Renaud et son hôte viennent de terminer leur dîner, et à la fin du récit Renaud tire la leçon de son exemple). Mais en même temps, et en dépit de cet encadrement à la manière de la nouvelle décaméronienne, le récit du chevalier est un récit autobiographique, qui le voit profondément impliqué et qu'il raconte plein de chagrin (XLIII 9, 3-4: vide abondare un gran rivo di pianto / dagli occhi del signor di quelle case). Loin de pouvoir consoler celui qui l'écoute, il a besoin de consolation, et, fait encore plus remarquable, il est moins sage que celui-ci, qui à la même occasion a fait preuve d'une plus grande prudence. La structure idéologique et fonctionnelle de la nouvelle se trouve complètement bouleversée, et ce qui la bouleverse est l'émergence

32. Tant par leur situation que par les premiers mots de leur récit, en effet, Astolphe et encore plus Lydie renvoient à des modèles dantesques (pour Astolphe notamment Pier delle Vigne), mais l'analogie apparaît aussi, bien que plus subtile, dans d'autres cas.

33. Dans la perception de la nouvelle au Cinquecento le ridicolo est en effet un trait essentiel de sa définition, comme on le voit dans le premier traité qui lui est consacré, la Lezione sopra il comporre delle novelle (I574) de Francesco Bonciani.

34. G. Sangirardi, Ludovico Ariosto, op. cit., p. 79-94 et p. II8-I28. 
du narrateur élégiaque, qui a moins du plaisir ou des leçons à offrir qu'une souffrance à exprimer. Or, dans ce narrateur élégiaque qu'est le chevalier de Mantoue on voit mieux que jamais le reflet autobiographique de l'Arioste : non seulement l'épisode se déroule entre Mantoue et Ferrare, mais le récit de la nouvelle est précédé par l'ekphrasis d'une fontaine en marbre située dans le palais du chevalier, qui célèbre prophétiquement les grandes dames des cours du temps de l'Arioste et les lettrés leurs amis. Parmi ces images, l'une reste sans nom, dans laquelle on devine le portrait de l'Arioste et de sa bien-aimée. Dans ce tissu de signes autobiographiques, le chevalier de Mantoue revêt ainsi avec une plus grande évidence le rôle de double élégiaque du poète. Son face-à-face avec Renaud, loin de représenter l'opposition entre la naïveté et la sagesse, comme on a pu le croire, articule la coexistence des deux voix, enfantine et adulte, dont joue incessamment la musique du roman. 